EGU2020-10979

https://doi.org/10.5194/egusphere-egu2020-10979

EGU General Assembly 2020

(c) Author(s) 2022. This work is distributed under

the Creative Commons Attribution 4.0 License.

\title{
Stable water isotopes as a tool to investigate tropospheric moisture transport pathways over the eastern subtropical North Atlantic
}

\author{
Fabienne Dahinden ${ }^{1}$, Franziska Aemisegger ${ }^{1}$, Sabine Barthlott ${ }^{2}$, Emanuel Christner ${ }^{2}$, Christoph \\ Dyroff $^{3}$, Frank Hase ${ }^{2}$, Peter Knippertz ${ }^{2}$, Heini Wernli ${ }^{1}$, Matthias Schneider ${ }^{2}$, and Stephan Pfahl ${ }^{4}$ \\ ${ }^{1}$ Institute for Atmospheric and Climate Science, ETH Zürich, Zürich, Switzerland \\ ${ }^{2}$ Institute of Meteorology and Climate Research, Karlsruhe Institute of Technology, Karlsruhe, Germany \\ ${ }^{3}$ Aerodyne Research Inc., 45 Manning Road, Billerica, MA 01821, USA \\ ${ }^{4}$ Institute for Meteorology, Freie Universität Berlin, Berlin, Germany
}

The subtropical atmospheric water cycle is a key component in the climate system. Freetropospheric humidity and low-level cloud cover over the subtropical oceans strongly affect the global radiative balance via the greenhouse and albedo effects. However, the complex interaction of dynamical processes controlling the subtropical tropospheric moisture budget is still not fully understood. Stable water isotopes have proven to be highly useful to investigate the physical mechanisms involved in the atmospheric water cycle. These natural tracers of water phase changes capture the moist diabatic history experienced by air parcels. Additionally, due to the distinct fingerprints of air masses with different origin, the isotopic composition of water vapor can further provide information about atmospheric processes that do not involve phase changes, for instance, turbulent mixing or large-scale water vapor transport. To enhance the understanding of the mechanisms controlling the subtropical tropospheric humidity, we performed dedicated high-resolution simulations with the isotope-enabled regional weather and climate prediction model COSMOiso. Comparison with ground-based remote sensing (Fourier transform infrared spectroscopy) and aircraft-based in situ isotope observations from the project MUSICA enables us to evaluate and constrain the representation of relevant physical processes in the model.

Our simulations confirm the current state of knowledge about the contrasting moisture transport conditions over the eastern subtropical North Atlantic, resulting from an interplay between humid, isotopically enriched air primarily coming from Africa on the one hand and dry, depleted air mainly originating from the upper-level extratropical North Atlantic on the other hand. Additionally, we show that North African air masses that are affected by the Saharan heat low (SHL) and air masses which come from the Sahel region further south are associated with a distinct isotope signature. This difference is mainly due to the fact that air masses from the Sahel region have experienced moist convection and cloud processing, whereas the Saharan air layer is a well-mixed air mass with a more homogenous isotope composition. We systematically assess the dynamical drivers 
behind these contrasting conditions. In particular, we investigate the importance of the SHL dynamics on moistening the free troposphere over the eastern subtropical North Atlantic. In summer, the SHL induces low-level convergence of air masses from different sources, which are then convectively lifted to higher altitudes and are eventually transported within the Saharan air layer across the North Atlantic, where they mix with dry, descending free tropospheric air. Detailed analysis of isotopic signals along kinematic back- trajectories of different air masses arriving over the Canary Islands allows to disentangle governing physical processes and relevant moisture sources that affect the free tropospheric humidity. The adopted Lagrangian isotope perspective notably enhances our understanding of air mass mixing and offers a sound interpretation of the free tropospheric humidity and isotopic variability on time scales of hours to days in contrasting atmospheric conditions over the eastern subtropical North Atlantic. 